\title{
The University and the Community: Reports on an extension project for TMD treatment by using laser therapy
}

\author{
Mariana Marquezan* \\ Universidade Federal de Santa Maria (UFSM), Santa Maria, Rio Grande do Sul, Brazil
}

Temporomandibular joint dysfunction (TMD) is characterized by a set of disturbances that involve the masticatory muscles, temporomandibular joints and associated structures, and is considered the most common cause of non-dental pain in the orofacial region. It affects approximately 30 to $44 \%$ of the population, and is more prevalent between 15 and 45 years of age, without distinction between the sexes. Approximately $16 \%$ of the population, however, require treatment, because many cases present self-resolution [1]. The etiology of TMD is multifactorial, involving physiopathological and anatomic factors; psychosocial aspects (psychiatric disease and emotional stress); and macro or micro traumas (including occlusal interferences, nocturnal bruxism, physical overload of the muscles) [2].

The scientific literature has shown us that reversible and conservative approaches must always be the first treatment option [3], and the following types may be cited: education (patients are made aware of their condition); self-care (stretching, exercises, changes in eating habits, sleep hygiene); psychological treatment; myorelaxant plate; drug therapy; botulinum toxin and laser therapy. These treatment modalities may be used alone or in association, according to the characteristics of each case. Secondarily, irreversible approaches such as orthodontic treatment; occlusal adjustments and surgical interventions may also be used [4]. Low level laser (LLL) has been used for the treatment of TMD because of its analgesic [5] and anti-inflammatory effect, and for the muscle relaxation promoted [6] by reducing fatigue and increasing bite force.

We understand that the University's mission goes beyond the production of scientific knowledge, because it must play an important role within the community in which it is inserted. In this context, university extension intends taking the scientific product of the University to the community. Based on the foregoing, we have created an extension project to offer treatment to the community affected by TMD, with laser therapy as the main treatment modality. The treatment proposed consisted of 10 sessions of laser therapy associated with verbal instructions on self-care, given in the first session, such as do not: chew gum, bite nails, lips or objects; consume stimulating foods and/or beverages after 19:00 hrs, and do perform sleep hygiene. An infrared laser tip (AsGaAl, $\lambda=808 \mathrm{~nm}$ ) was used with power of 100 $\mathrm{mW}$, in continuous mode, applying a fluence of $80 \mathrm{~J} / \mathrm{cm}^{2}$ on the TMJ musculature for 22 second per point (manufacturer's recommendation). The choice of LLLT application on the musculature was individualized for each patient, with the points sensitive to palpation being identified by means of the RDC/TMD (Research Diagnostic Criteria for Temporomandibular Disorders) criteria [7]. When there was pain in the TMJ, five points were irradiated: one on the TMJ; one superior; one inferior; one anterior, and one posterior point (within the external acoustic meatus). Two weekly sessions were performed for 5 weeks. If no improvement was observed with the use of LLLT, other therapeutic modalities were associated with it.

In the period of 12 months of existence of the project, 21 patients were treated; all presented with TMD diagnosed by the RDC, and had associated muscular and articular problems. Five of them presented with complete remission of symptoms after the proposed laser therapy sessions, without the need for association with other therapies. Another two patients had been using a myorelaxant plate when they entered the extension project, and obtained remission of the painful symptoms when the plate was associated with LLLT. Twelve patients received indication for use of the myorelaxant plate associated with LLLT, because they continued to feel pain after the irradiations proposed, in spite of observing improvement immediately after the irradiations. In these patients, nocturnal bruxism probably was a factor with great influence on the etiology of TMD, because the all reported rigidity in the face on awakening. As we knew that LLLT does not act on the factor bruxism, we indicated use of the myorelaxant plate associated with it. Finally, two patients discontinued the treatment.

It should be pointed out here that one patient, who sought treatment, presented with mouth opening so reduced that it made it unfeasible to fabricate the myorelaxant plate. Even with medication therapy, the patient was unable to increase his/her mouth opening. Only after the laser therapy sessions was it possible to increase the mouth opening, thus making it possible to take the patient's impressions for the purpose of fabricating the myorelaxant plate.

During the course of carrying out this project, the authors were able to confirm that LLLT was an important tool for controlling the painful symptoms and improving the functions of the stomatognathic system of patients with TMD. However, its application alone may not be effective when the etiological factors are active, so that the association of therapies would be necessary. Moreover, the authors perceived that the education of patients in changing their habits and self-care was

Correspondence to: Mariana Marquezan, Professor, Universidade Federa de Santa Maria (UFSM), Santa Maria, Rio Grande do Sul, Brazil, E-mail: marianamarquezan@gmail.com

Received: October 18, 2016; Accepted: October 28, 2016; Published: October 31,2016 
an important adjuvant to therapy. In addition, they recommend the elaboration of written instructions to help the patient remember these recommendations.

All the patients who participated in the project were invited to fill out a satisfaction diagram concerning the treatment performed, which showed $100 \%$ satisfaction, without any suggestion or criticism being recorded. The students involved in the project indicated in their statements that it had been extremely advantageous to have participated in this work, as they had learned more about the topics of TMD and laser therapy, and had experienced the satisfaction of helping people by reducing their pain and improving their masticatory function.

\section{References}

1. Al-Jundi MA, John MT, Setz JM, Szentpétery A, Kuss O (2008) Meta-analysis of treatment need for temporomandibular disorders in adult nonpatients. J Orofac Pain 22: 97-107.[Crossref]
2. McNeill C (1997) Management of temporomandibular disorders: concepts and controversies. J Prosthet Dent 77: 510-522. [Crossref]

3. Koyano K (2009)Temporomandibular disorders. Int J Prosthodont 22: 525-526. [Crossref]

4. Durham J, Newton-John TR, Zakrzewska JM (2015)Temporomandibular disorders BMJ 350: h1154.[Crossref]

5. Cetiner S, Kahraman SA, Yücetas S (2006) Evaluation of low-level laser therapy in the treatment of temporomandibular disorders. Photomed Laser Surg 24: 637-641. [Crossref]

6. Shinozaki EB, dos Santos MBF, Okazaki LK, Marchini L, Junior LB (2010) Clinical assessment of the efficacy of low-level laser therapy on muscle pain in women with temporomandibular dysfunction, by surface electromyography. Brazilian Journal of Oral Sciences 9: 434-438.

7. Pereira TS, Flecha OD, Guimarães RC, de Oliveira D, Botelho AM, et al.(2014) Efficacy of red and infrared lasers in treatment of temporomandibular disorders--a double-blind, randomized, parallel clinical trial. Cranio 32: 51-56.[Crossref]

Copyright: $@ 2016$ Marquezan M. This is an open-access article distributed under the terms of the Creative Commons Attribution License, which permits unrestricted use, distribution, and reproduction in any medium, provided the original author and source are credited. 